Supplement of Biogeosciences, 17, 381-404, 2020

https://doi.org/10.5194/bg-17-381-2020-supplement

(C) Author(s) 2020. This work is distributed under

the Creative Commons Attribution 4.0 License.

(c) (1)

Supplement of

\title{
Trace element composition of size-fractionated suspended particulate mat- ter samples from the Qatari Exclusive Economic Zone of the Arabian Gulf: the role of atmospheric dust
}

Oguz Yigiterhan et al.

Correspondence to: Oguz Yigiterhan (oguz@qu.edu.qa)

The copyright of individual parts of the supplement might differ from the CC BY 4.0 License. 


\section{Supplemental Materials:}

Table S1 (next three pages): Data for the trace element composition of plankton from the 2012 cruise with average leached Qatari dust used for the lithogenic correction. The upper segment, results for bulk plankton collected with a $50 \mu \mathrm{m}$ net tow, is separated from the lower segment showing data for the larger plankton collected with a $200 \mu \mathrm{m}$ net tow. The 'CUMULATIVE' row is an average of both of these measurements. The distance column gives the shortest distance from land to each sampling location (in kilometers). The letters (R), (L), and (E) represent the values for raw data, the lithogenic correction (in this case with average leached Qatari dust), and the excess concentrations, respectively. All are measured in parts per million $(\mathrm{ppm})$, or $\mu \mathrm{g} / \mathrm{g}$. Aluminum only has a raw data column because all of the aluminum in each sample was assumed to be of lithogenic origin, meaning that the lithogenic correction would be zero. The number in scientific notation in parenthesis adjacent to the $(\mathrm{L})$ column is a numerical representation of the [Me]/Al ratio found in Qatari dust (Table 1 and Yigiterhan et al, 2018) used to determine the lithogenic correction. Certain elements have excess values, which are negative, indicating that there was an overcorrection for the lithogenic portion of the sample. 


\begin{tabular}{|c|c|c|c|c|c|c|c|c|c|c|c|c|c|}
\hline Sample \# & Location & Distance (km) & Measurement & Al & As & As & As & $\mathbf{B a}$ & $\mathbf{B a}$ & $\mathbf{B a}$ & Cd & Cd & Cd \\
\hline & & & & $R$ & $R$ & $L(1.63 E-04)$ & $E$ & $R$ & $L(1.09 E-02)$ & $E$ & $R$ & $L(5.48 E-06)$ & $E$ \\
\hline 1 & Doha & 10.19 & Bulk $(50 \mu \mathrm{m})$ & 2,590 & 31.4 & 0.42 & 31.0 & 14.1 & 28.1 & -14.1 & 0.52 & 0.01 & 0.51 \\
\hline 2 & Khor Al-Odaid & 0.23 & Bulk $(50 \mu \mathrm{m})$ & 994 & 13.2 & 0.16 & 13.1 & 5.69 & 10.8 & -5.10 & 1.01 & 0.005 & 1.01 \\
\hline 3 & Mesaieed & 0.60 & Bulk $(50 \mu \mathrm{m})$ & 3,010 & 8.58 & 0.49 & 8.09 & 17.9 & 32.7 & -14.8 & 0.64 & 0.02 & 0.62 \\
\hline 4 & Shraawoo & 59.60 & Bulk $(50 \mu \mathrm{m})$ & 1,940 & 13.9 & 0.32 & 13.6 & 14.7 & 21.1 & -6.39 & 1.11 & 0.01 & 1.10 \\
\hline 5 & Al-Edd Al-Gharbi & 46.55 & Bulk $(50 \mu \mathrm{m})$ & 480 & 6.57 & 0.08 & 6.49 & 53.4 & 5.21 & 48.1 & 0.81 & 0.003 & 0.81 \\
\hline 6 & Ash Shargi Oilfield & 78.90 & Bulk $(50 \mu \mathrm{m})$ & 1,830 & 9.19 & 0.30 & 8.89 & 26.8 & 19.9 & 6.89 & 2.71 & 0.01 & 2.70 \\
\hline 7 & Halul Island & 84.05 & Bulk $(50 \mu \mathrm{m})$ & 1,030 & 10.9 & 0.17 & 10.7 & 12.4 & 11.1 & 1.25 & 3.59 & 0.01 & 3.58 \\
\hline 8 & High North & 74.55 & Bulk $(50 \mu \mathrm{m})$ & 1,180 & 12.2 & 0.19 & 12.0 & 6.63 & 12.8 & -6.20 & 2.03 & 0.01 & 2.02 \\
\hline 9 & Ras Laffan & 11.40 & Bulk $(50 \mu \mathrm{m})$ & 803 & 16.1 & 0.13 & 16.0 & 5.55 & 8.72 & -3.17 & 1.56 & 0.004 & 1.56 \\
\hline 10 & Dukhan & 0.02 & Bulk $(50 \mu \mathrm{m})$ & 10,500 & 28.5 & 1.71 & 26.8 & 69.5 & 114 & -44.7 & 0.36 & 0.06 & 0.30 \\
\hline 11 & Umm Bab & 0.48 & Bulk $(50 \mu \mathrm{m})$ & 7,230 & 19.3 & 1.18 & 18.1 & 42.5 & 78.4 & -36.0 & 0.54 & 0.02 & 0.52 \\
\hline AVERAGE & & & & 2,870 & 15.4 & 0.468 & 15.0 & 24.5 & 31.2 & -6.73 & 1.35 & 0.016 & 1.34 \\
\hline
\end{tabular}

\begin{tabular}{|c|c|c|c|c|c|c|c|c|c|c|c|c|c|}
\hline & & & & $R$ & $R$ & $L(1.63 E-04)$ & $E$ & $R$ & $L(1.09 E-02)$ & $E$ & $R$ & $L(5.48 E-06)$ & $E$ \\
\hline 12 & Doha & 10.19 & Zooplankton $(200 \mu \mathrm{m})$ & 3,250 & 17.9 & 0.53 & 17.4 & 16.9 & 35.3 & -18.4 & 0.70 & 0.0178 & 0.686 \\
\hline 13 & Khor Al-Odaid & 0.23 & Zooplankton $(200 \mu \mathrm{m})$ & 1,010 & 8.36 & 0.16 & 8.19 & 6.07 & 11.0 & -4.91 & 1.17 & 0.0055 & 1.17 \\
\hline 14 & Mesaieed & 0.60 & Zooplankton $(200 \mu \mathrm{m})$ & 2,770 & 7.64 & 0.45 & 7.19 & 11.8 & 30.1 & -18.3 & 0.62 & 0.01 & 0.61 \\
\hline 15 & Shraawoo & 59.60 & Zooplankton $(200 \mu \mathrm{m})$ & 722 & 10.7 & 0.12 & 10.5 & 4.77 & 7.83 & -3.07 & 0.78 & 0.004 & 0.78 \\
\hline 16 & Al-Edd Al-Gharbi & 46.55 & Zooplankton $(200 \mu \mathrm{m})$ & 641 & 9.22 & 0.10 & 9.11 & 114 & 6.96 & 107 & 1.00 & 0.004 & 0.99 \\
\hline 17 & Ash Shargi Oilfield & 78.90 & Zooplankton $(200 \mu \mathrm{m})$ & 560 & 7.76 & 0.09 & 7.67 & 15.6 & 6.07 & 9.57 & 2.07 & 0.003 & 2.07 \\
\hline 18 & Halul Island & 84.05 & Zooplankton $(200 \mu \mathrm{m})$ & 335 & 12.1 & 0.05 & 12.0 & 12.8 & 3.63 & 9.15 & 2.74 & 0.002 & 2.74 \\
\hline 19 & High North & 74.55 & Zooplankton $(200 \mu \mathrm{m})$ & 1,020 & 14.5 & 0.17 & 14.3 & 6.68 & 11.1 & -4.38 & 2.27 & 0.01 & 2.26 \\
\hline 20 & Ras Laffan & 11.40 & Zooplankton $(200 \mu \mathrm{m})$ & 418 & 19.4 & 0.07 & 19.3 & 2.78 & 4.54 & -1.76 & 1.77 & 0.002 & 1.76 \\
\hline 21 & Dukhan & 0.02 & Zooplankton $(200 \mu \mathrm{m})$ & 5,560 & 19.5 & 0.90 & 18.6 & 37.6 & 60.3 & -22.8 & 0.59 & 0.03 & 0.56 \\
\hline 22 & Umm Bab & 0.48 & Zooplankton $(200 \mu \mathrm{m})$ & 1,870 & 9.46 & 0.30 & 9.15 & 17.5 & 20.2 & -2.77 & 0.43 & 0.01 & 0.42 \\
\hline AVERAGE & & & & 1,650 & 12.4 & 0.27 & 12.1 & 22.4 & 17.9 & 4.51 & 1.29 & 0.009 & 1.28 \\
\hline CUMULATIVE & & & & 2,260 & 13.9 & 0.37 & 13.6 & 23.4 & 24.6 & -1.11 & 1.32 & 0.012 & 1.31 \\
\hline
\end{tabular}




\begin{tabular}{|c|c|c|c|c|c|c|c|c|c|c|c|c|c|c|c|}
\hline Sample \# & Co & Co & Co & $\mathrm{Cr}$ & $\mathrm{Cr}$ & $\mathbf{C r}$ & $\mathbf{C u}$ & $\mathbf{C u}$ & $\mathrm{Cu}$ & $\mathbf{F e}$ & $\mathbf{F e}$ & $\mathbf{F e}$ & Mn & Mn & Mn \\
\hline & $R$ & $L(2.30 E-04)$ & $E$ & $R$ & $L(3.42 E-03)$ & $\boldsymbol{E}$ & $\boldsymbol{R}$ & $L(1.12 E-03)$ & $\boldsymbol{E}$ & $R$ & $L(6.06 E-01)$ & $E$ & $R$ & $L(6.74 E-03)$ & $\boldsymbol{E}$ \\
\hline 1 & 1.18 & 0.60 & 0.58 & 6.31 & 8.86 & -2.55 & 9.28 & 2.91 & 6.37 & 1,360 & 1,570 & -211 & 26.3 & 17.5 & 8.77 \\
\hline 2 & 1.00 & 0.23 & 0.77 & 3.72 & 3.40 & 0.32 & 10.4 & 1.12 & 9.26 & 793 & 602 & 191 & 52.2 & 6.70 & 45.5 \\
\hline 3 & 1.14 & 0.69 & 0.45 & 11.6 & 10.3 & 1.30 & 184 & 3.39 & 180 & 2,200 & 1,820 & 376 & 50.1 & 20.3 & 29.8 \\
\hline 4 & 0.96 & 0.45 & 0.51 & 5.21 & 6.64 & -1.42 & 82.3 & 2.18 & 80.2 & 1,020 & 1,180 & -154 & 35.5 & 13.1 & 22.4 \\
\hline 5 & 0.52 & 0.11 & 0.41 & 1.20 & 1.64 & -0.44 & 10.8 & 0.54 & 10.2 & 239 & 291 & -51.8 & 13.2 & 3.24 & 9.94 \\
\hline 6 & 0.96 & 0.42 & 0.53 & 6.40 & 6.25 & 0.15 & 41.4 & 2.06 & 39.4 & 977 & 1,110 & -131 & 31.7 & 12.3 & 19.3 \\
\hline 7 & 1.02 & 0.24 & 0.78 & 8.51 & 3.51 & 5.01 & 13.9 & 1.15 & 12.8 & 437 & 621 & -184 & 16.0 & 6.92 & 9.08 \\
\hline 8 & 0.88 & 0.27 & 0.61 & 3.71 & 4.04 & -0.33 & 11.2 & 1.33 & 9.89 & 526 & 716 & -190 & 21.5 & 7.98 & 13.5 \\
\hline 9 & 0.52 & 0.19 & 0.33 & 4.00 & 2.74 & 1.25 & 56.9 & 0.90 & 56.0 & 445 & 486 & -41.1 & 12.8 & 5.42 & 7.36 \\
\hline 10 & 3.00 & 2.42 & 0.58 & 24.9 & 36.0 & -11.1 & 11.9 & 11.8 & 0.04 & 5,490 & 6,370 & -879 & 133 & 71.0 & 61.7 \\
\hline 11 & 2.38 & 1.66 & 0.72 & 17.6 & 24.7 & -7.11 & 12.6 & 8.12 & 4.51 & 3,630 & 4,380 & -748 & 88.6 & 48.7 & 39.8 \\
\hline AVERAGE & 1.23 & 0.66 & 0.57 & 8.47 & 9.82 & -1.36 & 40.4 & 3.23 & 37.2 & 1,560 & 1,740 & -184 & 43.7 & 19.4 & 24.3 \\
\hline
\end{tabular}

\begin{tabular}{|c|c|c|c|c|c|c|c|c|c|c|c|c|c|c|c|}
\hline & $R$ & $L(2.30 E-04)$ & $E$ & $R$ & $L(3.42 E-03)$ & $E$ & $R$ & $L(1.12 E-03)$ & $E$ & $R$ & $L(6.06 E-01)$ & $E$ & $R$ & $L(6.74 E-03)$ & $E$ \\
\hline 12 & 1.17 & 0.75 & 0.42 & 8.96 & 11.1 & -2.15 & 12.9 & 3.66 & 9.23 & 1,770 & 1,970 & -199 & 37.2 & 21.9 & 15.3 \\
\hline 13 & 1.30 & 0.23 & 1.07 & 3.07 & 3.46 & -0.39 & 9.75 & 1.14 & 8.61 & 817 & 613 & 205 & 75.3 & 6.82 & 68.5 \\
\hline 14 & 1.93 & 0.64 & 1.29 & 11.9 & 9.48 & 2.39 & 14.1 & 3.12 & 10.9 & 2,000 & 1,680 & 322 & 57.1 & 18.7 & 38.4 \\
\hline 15 & 0.58 & 0.17 & 0.41 & 1.93 & 2.47 & -0.54 & 6.77 & 0.81 & 5.96 & 370 & 437 & -67.5 & 27.0 & 4.87 & 22.2 \\
\hline 16 & 0.68 & 0.15 & 0.53 & 2.40 & 2.19 & 0.21 & 35.5 & 0.72 & 34.8 & 315 & 388 & -73.0 & 20.3 & 4.32 & 15.9 \\
\hline 17 & 0.48 & 0.13 & 0.35 & 2.09 & 1.91 & 0.18 & 48.9 & 0.63 & 48.3 & 262 & 339 & -77.0 & 15.3 & 3.77 & 11.5 \\
\hline 18 & 0.43 & 0.07 & 0.36 & 2.65 & 1.14 & 1.51 & 12.7 & 0.38 & 12.3 & 136 & 203 & -66.4 & 5.69 & 2.26 & 3.43 \\
\hline 19 & 0.70 & 0.23 & 0.47 & 2.61 & 3.48 & -0.87 & 13.1 & 1.15 & 12.0 & 484 & 617 & -133 & 22.5 & 6.87 & 15.6 \\
\hline 20 & 0.39 & 0.10 & 0.29 & 3.29 & 1.43 & 1.87 & 14.1 & 0.47 & 13.6 & 222 & 253 & -31.4 & 7.74 & 2.82 & 4.91 \\
\hline 21 & 2.28 & 1.28 & 1.00 & 14.4 & 19.0 & -4.65 & 9.54 & 6.25 & 3.29 & 2,920 & 3,370 & -443 & 79.2 & 37.5 & 41.7 \\
\hline 22 & 1.39 & 0.43 & 0.96 & 4.62 & 6.37 & -1.76 & 8.75 & 2.10 & 6.65 & 988 & 1,130 & -141 & 82.5 & 12.6 & 69.9 \\
\hline AVERAGE & 1.03 & 0.38 & 0.65 & 5.26 & 5.64 & -0.38 & 16.9 & 1.86 & 15.1 & 935 & 1,000 & -64.2 & 39.1 & 11.1 & 27.9 \\
\hline CUMULATIVE & 1.13 & 0.52 & 0.61 & 6.9 & 7.73 & -0.87 & 28.7 & 2.54 & 26.1 & 1,250 & 1,370 & -124 & 41.4 & 15.3 & 26.1 \\
\hline
\end{tabular}




\begin{tabular}{|c|c|c|c|c|c|c|c|c|c|c|c|c|c|c|c|}
\hline Sample \# & Mo & Mo & Mo & $\mathrm{Ni}$ & $\mathrm{Ni}$ & $\mathbf{N i}$ & $\mathbf{P b}$ & $\mathbf{P b}$ & $\mathbf{P b}$ & $\mathbf{V}$ & $\mathbf{V}$ & $\mathbf{V}$ & $\mathbf{Z n}$ & $\mathbf{Z n}$ & $\mathbf{Z n}$ \\
\hline & $R$ & $L(1.20 E-03)$ & $E$ & $R$ & $L(4.91 E-03)$ & $E$ & $R$ & $L(2.10 E-04)$ & $E$ & $R$ & $L(1.80 E-03)$ & $E$ & $R$ & $L(4.93 E-03)$ & $E$ \\
\hline 1 & 117 & 3.11 & 114 & 5.99 & 12.7 & -6.73 & 0.70 & 0.54 & 0.16 & 4.59 & 4.66 & -0.06 & 25.5 & 12.8 & 12.7 \\
\hline 2 & 2.59 & 1.19 & 1.40 & 2.94 & 4.88 & -1.93 & 0.34 & 0.21 & 0.13 & 26.7 & 1.79 & 25.0 & 78.9 & 4.90 & 74.0 \\
\hline 3 & 9.26 & 3.61 & 5.65 & 7.71 & 14.8 & -7.07 & 3.01 & 0.63 & 2.38 & 18.7 & 5.41 & 13.3 & 185 & 14.9 & 170 \\
\hline 4 & 17.6 & 2.33 & 15.2 & 4.77 & 9.53 & -4.76 & 0.50 & 0.41 & 0.09 & 6.62 & 3.49 & 3.13 & 89.8 & 9.58 & 80.3 \\
\hline 5 & 0.00 & 0.58 & -0.58 & 1.17 & 2.36 & -1.19 & 0.33 & 0.10 & 0.23 & 1.27 & 0.86 & 0.40 & 24.5 & 2.37 & 22.2 \\
\hline 6 & 0.31 & 2.19 & -1.88 & 5.27 & 8.98 & -3.71 & 0.80 & 0.38 & 0.42 & 5.27 & 3.29 & 1.98 & 68.7 & 9.03 & 59.6 \\
\hline 7 & 0.27 & 1.23 & -0.96 & 5.20 & 5.04 & 0.16 & 0.19 & 0.23 & -0.03 & 2.05 & 1.84 & 0.20 & 73.7 & 5.06 & 68.6 \\
\hline 8 & 0.00 & 1.42 & -1.42 & 4.26 & 5.80 & -1.54 & 0.31 & 0.25 & 0.06 & 1.97 & 2.12 & -0.15 & 36.6 & 5.83 & 30.7 \\
\hline 9 & 0.34 & 0.96 & -0.62 & 3.12 & 3.94 & -0.82 & 0.80 & 0.17 & 0.63 & 2.08 & 1.44 & 0.64 & 98.8 & 3.96 & 94.8 \\
\hline 10 & 0.18 & 12.60 & -12.4 & 21.1 & 51.6 & -30.6 & 3.03 & 2.21 & 0.82 & 27.3 & 18.9 & 8.41 & 48.5 & 51.9 & -3.43 \\
\hline 11 & 0.32 & 8.66 & -8.34 & 14.1 & 35.5 & -21.3 & 2.12 & 1.52 & 0.60 & 19.3 & 13.0 & 6.30 & 41.7 & 35.7 & 6.02 \\
\hline AVERAGE & 13.4 & 3.44 & 9.98 & 6.87 & 14.1 & -7.23 & 1.10 & 0.60 & 0.50 & 10.5 & 5.16 & 5.37 & 70.1 & 14.2 & 55.9 \\
\hline
\end{tabular}

\begin{tabular}{|c|c|c|c|c|c|c|c|c|c|c|c|c|c|c|c|}
\hline & $R$ & $L(1.20 E-03)$ & $E$ & $\boldsymbol{R}$ & $L(4.91 E-03)$ & $E$ & $R$ & $L(2.10 E-04)$ & $E$ & $R$ & $L(1.80 E-03)$ & $\boldsymbol{E}$ & $R$ & $L(4.93 E-03)$ & $\boldsymbol{E}$ \\
\hline 12 & 66.1 & 3.90 & 62.3 & 7.30 & 16.0 & -8.65 & 1.15 & 0.68 & 0.461 & 6.50 & 5.84 & 0.65 & 49.2 & 16.0 & 33.2 \\
\hline 13 & 2.98 & 1.21 & 1.76 & 3.15 & 4.96 & -1.81 & 0.18 & 0.21 & -0.03 & 10.5 & 1.82 & 8.65 & 90.1 & 4.99 & 85.1 \\
\hline 14 & 17.6 & 3.32 & 14.3 & 7.57 & 13.6 & -6.04 & 2.03 & 0.58 & 1.44 & 9.69 & 4.98 & 4.71 & 74.6 & 13.7 & 60.9 \\
\hline 15 & 17.7 & 0.87 & 16.9 & 1.73 & 3.54 & -1.81 & 0.16 & 0.15 & 0.01 & 2.99 & 1.30 & 1.69 & 42.3 & 3.56 & 38.7 \\
\hline 16 & 0.00 & 0.77 & -0.77 & 1.58 & 3.15 & -1.57 & 0.56 & 0.13 & 0.43 & 1.80 & 1.15 & 0.645 & 46.7 & 3.16 & 43.5 \\
\hline 17 & 0.00 & 0.67 & -0.67 & 1.55 & 2.74 & -1.19 & 0.00 & 0.12 & -0.12 & 1.88 & 1.01 & 0.878 & 61.7 & 2.76 & 59.0 \\
\hline 18 & 0.10 & 0.40 & -0.30 & 2.03 & 1.64 & 0.39 & 0.14 & 0.07 & 0.07 & 0.91 & 0.60 & 0.31 & 64.9 & 1.65 & 63.3 \\
\hline 19 & 0.00 & 1.22 & -1.22 & 3.90 & 5.00 & -1.10 & 0.57 & 0.21 & 0.35 & 2.17 & 1.83 & 0.34 & 45.9 & 5.03 & 40.9 \\
\hline 20 & 0.98 & 0.50 & 0.48 & 2.80 & 2.05 & 0.748 & 0.00 & 0.09 & -0.09 & 1.22 & 0.75 & 0.47 & 80.6 & 2.06 & 78.6 \\
\hline 21 & 0.00 & 6.66 & -6.66 & 11.6 & 27.3 & -15.70 & 1.58 & 1.17 & 0.41 & 14.7 & 9.99 & 4.68 & 67.3 & 27.4 & 39.9 \\
\hline 22 & 0.00 & 2.24 & -2.24 & 3.98 & 9.15 & -5.17 & 0.89 & 0.39 & 0.50 & 5.30 & 3.35 & 1.95 & 41.8 & 9.20 & 32.6 \\
\hline AVERAGE & 9.60 & 1.98 & 7.62 & 4.29 & 8.10 & -3.81 & 0.66 & 0.35 & 0.31 & 5.24 & 2.97 & 2.27 & 60.5 & 8.14 & 52.3 \\
\hline CUMULATIVE & 11.5 & 2.71 & 8.80 & 5.58 & 11.1 & -5.52 & 0.88 & 0.48 & 0.40 & 7.89 & 4.06 & 3.82 & 65.3 & 11.2 & 54.1 \\
\hline
\end{tabular}


Table S2 (next three pages): Data for the trace element composition of plankton from the 2014 cruise with average leached Qatari dust used for the lithogenic correction. The upper segment, results for bulk plankton collected with a $50 \mu \mathrm{m}$ net tow, is separated from the lower segment showing data for the larger plankton collected with a $200 \mu \mathrm{m}$ net tow. The 'CUMULATIVE' row is an average of both of these measurements. The distance column gives the shortest distance from land to each sampling location (in kilometers). The letters (R), (L), and (E) represent the values for raw data, the lithogenic correction (in this case with average leached Qatari dust), and the excess concentrations, respectively. All are measured in parts per million (ppm), or $\mu \mathrm{g} / \mathrm{g}$. Aluminum only has a raw data column because all of the aluminum in each sample was assumed to be of lithogenic origin, meaning that the lithogenic correction would be zero. The number in scientific notation in parenthesis adjacent to the (L) column is a numerical representation of the [Me]/Al ratio found in Qatari dust (Yigiterhan et al, in preparation) used to determine the lithogenic correction. Certain elements have excess values which are negative, indicating that there was an overcorrection for the lithogenic portion of the sample. 


\begin{tabular}{|c|c|c|c|c|c|c|c|c|c|c|c|c|c|}
\hline & & & & $\mathrm{R}$ & $\mathrm{R}$ & L (1.63E-04) & E & $\mathrm{R}$ & L (1.09E-02) & $\mathrm{E}$ & $\mathrm{R}$ & L (5.48E-06) & $\mathrm{E}$ \\
\hline Sample & Location & Distance (km) & Measurement & Al & As & As & As & Ba & $\mathbf{B a}$ & $\mathbf{B a}$ & Cd & Cd & Cd \\
\hline 23 & Dukhan & 8.77 & Bulk $(50 \mu \mathrm{m})$ & 4,190 & 8.72 & 0.68 & 8.04 & 22.9 & 45.4 & -22.6 & 0.84 & 0.02 & 0.81 \\
\hline 24 & Dukhan & 8.77 & Bulk $(50 \mu \mathrm{m})$ & 4,070 & 8.07 & 0.66 & 7.41 & 21.0 & 44.2 & -23.2 & 0.76 & 0.02 & 0.74 \\
\hline 25 & Dukhan & 6.78 & Bulk $(50 \mu \mathrm{m})$ & 3,950 & 9.72 & 0.64 & 9.07 & 22.1 & 42.9 & -20.8 & 0.79 & 0.02 & 0.77 \\
\hline 26 & Dukhan & 6.78 & Bulk $(50 \mu \mathrm{m})$ & 4,110 & 9.51 & 0.67 & 8.84 & 21.2 & 44.7 & -23.5 & 0.80 & 0.02 & 0.77 \\
\hline 27 & Dukhan & 1.31 & Bulk $(50 \mu \mathrm{m})$ & 8,480 & 14.9 & 1.38 & 13.5 & 43.5 & 92.1 & -48.6 & 0.19 & 0.05 & 0.14 \\
\hline 28 & Dukhan & 1.31 & Bulk $(50 \mu \mathrm{m})$ & 8,330 & 14.0 & 1.36 & 12.7 & 40.9 & 90.4 & -49.5 & 0.19 & 0.05 & 0.15 \\
\hline 29 & Doha & 6.54 & Bulk $(50 \mu \mathrm{m})$ & 4,400 & 3.87 & 0.72 & 3.15 & 28.6 & 47.8 & -19.2 & 0.26 & 0.02 & 0.24 \\
\hline 30 & Doha & 2.20 & Bulk $(50 \mu \mathrm{m})$ & 3,380 & 2.04 & 0.55 & 1.49 & 13.0 & 36.7 & -23.7 & 0.08 & 0.02 & 0.06 \\
\hline 31 & Doha & 2.20 & Bulk $(50 \mu \mathrm{m})$ & 3,150 & 1.97 & 0.51 & 1.46 & 12.1 & 34.2 & -22.1 & 0.09 & 0.02 & 0.07 \\
\hline 32 & Doha & 0.06 & Bulk $(50 \mu \mathrm{m})$ & 14,400 & 4.43 & 2.34 & 2.09 & 65.6 & 156 & -90.4 & 0.12 & 0.08 & 0.04 \\
\hline 33 & Doha & 0.06 & Bulk $(50 \mu \mathrm{m})$ & 14,600 & 4.66 & 2.38 & 2.28 & 73.4 & 159 & -85.3 & 0.09 & 0.08 & 0.01 \\
\hline AVERAGE & & & & 6,640 & 7.44 & 1.08 & 6.36 & 33.1 & 72.1 & -39.0 & 0.38 & 0.04 & 0.35 \\
\hline
\end{tabular}

\begin{tabular}{|c|c|c|c|c|c|c|c|c|c|c|c|c|c|}
\hline & & & & Al & As & As & As & $\mathbf{B a}$ & $\mathbf{B a}$ & Ba & Cd & Cd & Cd \\
\hline 34 & Dukhan & 8.77 & Zooplankton $(200 \mu \mathrm{m})$ & 2,050 & 7.04 & 0.33 & 6.70 & 14.1 & 22.3 & -8.16 & 0.77 & 0.01 & 0.75 \\
\hline 35 & Dukhan & 6.78 & Zooplankton $(200 \mu \mathrm{m})$ & 4,450 & 5.83 & 0.72 & 5.10 & 78.4 & 48.3 & 30.1 & 0.29 & 0.02 & 0.27 \\
\hline 36 & Dukhan & 1.31 & Zooplankton $(200 \mu \mathrm{m})$ & 4,190 & 5.17 & 0.68 & 4.49 & 33.9 & 45.5 & -11.6 & 0.12 & 0.02 & 0.10 \\
\hline 37 & Doha & 6.54 & Zooplankton $(200 \mu \mathrm{m})$ & 3,730 & 4.10 & 0.61 & 3.49 & 22.0 & 40.5 & -18.5 & 0.62 & 0.02 & 0.60 \\
\hline 38 & Doha & 2.20 & Zooplankton $(200 \mu \mathrm{m})$ & 7,630 & 2.45 & 1.24 & 1.20 & 40.9 & 82.9 & -42.0 & 0.37 & 0.04 & 0.32 \\
\hline 39 & Doha & 0.06 & Zooplankton $(200 \mu \mathrm{m})$ & 5,750 & 5.10 & 0.94 & 4.17 & 24.6 & 62.4 & -37.9 & 0.31 & 0.03 & 0.28 \\
\hline 40 & Doha & 0.06 & Zooplankton $(200 \mu \mathrm{m})$ & 5,260 & 4.62 & 0.86 & 3.76 & 25.1 & 57.1 & -32.1 & 0.34 & 0.03 & 0.31 \\
\hline AVERAGE & & & & 4,720 & 4.90 & 0.769 & 4.13 & 34.1 & 51.3 & -17.1 & 0.40 & 0.03 & 0.38 \\
\hline
\end{tabular}

\begin{tabular}{|l|l|l|l|l|l|l|l|l|l|l|l|l|l|}
\hline CUMULATIVE & & & & 5,900 & $\mathbf{6 . 4 6}$ & $\mathbf{0 . 9 6 0}$ & $\mathbf{5 . 5 0}$ & $\mathbf{3 3 . 5}$ & $\mathbf{6 4 . 0}$ & $\mathbf{- 3 0 . 5}$ & $\mathbf{0 . 3 9}$ & $\mathbf{0 . 0 3}$ & $\mathbf{0 . 3 6}$ \\
\hline
\end{tabular}




\begin{tabular}{|c|c|c|c|c|c|c|c|c|c|c|c|c|c|c|c|}
\hline & $\mathrm{R}$ & L (2.30E-04) & E & $\mathrm{R}$ & L (3.42E-03) & E & $\mathrm{R}$ & L (1.12E-03) & E & $\mathrm{R}$ & L (6.06E-01) & E & $\mathrm{R}$ & L (6.74E-03) & $\mathrm{E}$ \\
\hline Sample & Co & Co & Co & $\mathrm{Cr}$ & $\mathrm{Cr}$ & $\mathrm{Cr}$ & $\mathrm{Cu}$ & $\mathrm{Cu}$ & $\mathbf{C u}$ & $\mathrm{Fe}$ & $\mathbf{F e}$ & $\mathbf{F e}$ & Mn & Mn & Mn \\
\hline 23 & 1.84 & 0.96 & 0.88 & 9.86 & 14.3 & -4.45 & 28.4 & 4.71 & 23.6 & 2,520 & 2,540 & -12.4 & 45.7 & 28.2 & 17.4 \\
\hline 24 & 1.70 & 0.94 & 0.77 & 10.3 & 13.9 & -3.64 & 43.6 & 4.58 & 39.1 & 2,190 & 2,460 & -271 & 42.5 & 27.5 & 15.1 \\
\hline 25 & 1.69 & 0.91 & 0.78 & 8.76 & 13.5 & -4.76 & 65.3 & 4.45 & 60.9 & 2,300 & 2,390 & -90 & 43.6 & 26.7 & 16.9 \\
\hline 26 & 1.67 & 0.95 & 0.72 & 8.92 & 14.1 & -5.14 & 65.1 & 4.63 & 60.5 & 2,110 & 2,490 & -378 & 42.6 & 27.8 & 14.9 \\
\hline 27 & 2.68 & 1.95 & 0.73 & 17.1 & 29.0 & -11.9 & 17.2 & 9.54 & 7.70 & 4,430 & 5,140 & -701 & 70.9 & 57.2 & 13.7 \\
\hline 28 & 2.56 & 1.92 & 0.64 & 16.3 & 28.5 & -12.1 & 26.1 & 9.36 & 16.7 & 4,080 & 5,040 & -962 & 66.0 & 56.2 & 9.83 \\
\hline 29 & 1.78 & 1.01 & 0.77 & 15.0 & 15.0 & -0.08 & 311 & 4.95 & 306 & 2,600 & 2,660 & -62 & 40.1 & 29.7 & 10.4 \\
\hline 30 & 1.44 & 0.78 & 0.66 & 9.43 & 11.5 & -2.12 & 26.1 & 3.80 & 22.3 & 1810 & 2,050 & -240 & 24.3 & 22.8 & 1.50 \\
\hline 31 & 1.38 & 0.73 & 0.66 & 8.31 & 10.8 & -2.47 & 63.5 & 3.55 & 60.0 & 1,700 & 1,910 & -211 & 22.5 & 21.3 & 1.24 \\
\hline 32 & 3.74 & 3.31 & 0.43 & 42.9 & 49.1 & -6.24 & 37.8 & 16.2 & 21.6 & 7,020 & 8,700 & $-1,680$ & 147 & 96.9 & 50.5 \\
\hline 33 & 3.97 & 3.37 & 0.61 & 45.1 & 49.9 & -4.83 & 34.0 & 16.4 & 17.6 & 7,390 & 8,850 & $-1,460$ & 153 & 98.6 & 54.5 \\
\hline AVERAGE & 2.22 & 1.53 & 0.69 & 17.4 & 22.7 & -5.25 & 65.3 & 7.47 & 57.8 & 3,470 & 4,020 & -552 & 63.5 & 44.8 & 18.7 \\
\hline
\end{tabular}

\begin{tabular}{|c|c|c|c|c|c|c|c|c|c|c|c|c|c|c|c|}
\hline & Co & Co & Co & $\mathrm{Cr}$ & $\mathrm{Cr}$ & $\mathrm{Cr}$ & $\mathbf{C u}$ & $\mathrm{Cu}$ & $\mathbf{C u}$ & $\mathrm{Fe}$ & $\mathrm{Fe}$ & $\mathrm{Fe}$ & Mn & Mn & Mn \\
\hline 34 & 2.45 & 0.47 & 1.98 & -27.2 & 7.02 & -34.2 & 31.9 & 2.31 & 29.6 & 1,760 & 1,240 & 513 & 45.8 & 13.9 & 32.0 \\
\hline 35 & 1.75 & 1.02 & 0.72 & 17.5 & 15.2 & 2.28 & 6.87 & 5.00 & 1.87 & 3,280 & 2,690 & 582 & 83.4 & 30.0 & 53.4 \\
\hline 36 & 2.15 & 0.97 & 1.19 & 12.6 & 14.3 & -1.74 & 125 & 4.71 & 120 & 3,300 & 2,540 & 762 & 70.3 & 28.3 & 42.0 \\
\hline 37 & 1.79 & 0.86 & 0.93 & 12.7 & 12.7 & -0.01 & 368 & 4.19 & 363 & 2,870 & 2,260 & 617 & 45.6 & 25.1 & 20.4 \\
\hline 38 & 4.25 & 1.76 & 2.49 & 44.4 & 26.1 & 18.3 & 28 & 8.58 & 19.4 & 5,930 & 4,620 & 1,310 & 184 & 51.5 & 133 \\
\hline 39 & 4.50 & 1.32 & 3.18 & 25.1 & 19.7 & 5.48 & 26.8 & 6.47 & 20.4 & 3,540 & 3,480 & 54.3 & 494 & 38.8 & 455 \\
\hline 40 & 4.18 & 1.21 & 2.97 & 22.2 & 18.0 & 4.22 & 32.2 & 5.91 & 26.3 & 3,330 & 3,190 & 145 & 471 & 35.5 & 435 \\
\hline AVERAGE & 3.01 & 1.09 & 1.92 & 15.3 & 16.1 & -0.82 & 88.3 & 5.31 & 83.0 & $3, \mathbf{4 3 0}$ & 2,860 & 569 & 199 & 31.9 & 167 \\
\hline
\end{tabular}

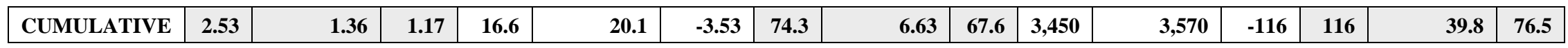




\begin{tabular}{|c|c|c|c|c|c|c|c|c|c|c|c|c|c|c|c|}
\hline & $\mathrm{R}$ & L (1.20E-03) & $\mathrm{E}$ & $\mathrm{R}$ & L (4.91E-03) & $\mathrm{E}$ & $\mathrm{R}$ & L (2.10E-04) & E & $\mathrm{R}$ & $\mathrm{L}(1.80 \mathrm{E}-03)$ & $\mathrm{E}$ & $\mathrm{R}$ & L (4.93E-03) & E \\
\hline Sample & Mo & Mo & Mo & $\mathbf{N i}$ & $\mathbf{N i}$ & $\mathrm{Ni}$ & $\mathbf{P b}$ & $\mathbf{P b}$ & $\mathbf{P b}$ & $\mathbf{V}$ & $\mathbf{V}$ & $\mathbf{V}$ & Zn & Zn & Zn \\
\hline 23 & 0.27 & 5.02 & -4.75 & 10.2 & 20.5 & -10.4 & 1.55 & 0.88 & 0.67 & 17.8 & 7.52 & 10.3 & 111 & 20.7 & 90.4 \\
\hline 24 & 0.29 & 4.88 & -4.59 & 9.27 & 20.0 & -10.7 & 1.05 & 0.86 & 0.19 & 16.1 & 7.31 & 8.83 & 120 & 20.1 & 100 \\
\hline 25 & 0.07 & 4.74 & -4.67 & 9.27 & 19.4 & -10.1 & 1.21 & 0.83 & 0.38 & 16.8 & 7.10 & 9.66 & 78.3 & 19.5 & 58.8 \\
\hline 26 & 0.22 & 4.93 & -4.71 & 9.14 & 20.2 & -11.0 & 5.53 & 0.87 & 4.67 & 16.6 & 7.39 & 9.25 & 75.3 & 20.3 & 55.0 \\
\hline 27 & -0.06 & 10.2 & -10.2 & 16.1 & 41.6 & -25.5 & 2.90 & 1.78 & 1.12 & 21.8 & 15.2 & 6.61 & 30.1 & 41.8 & -11.7 \\
\hline 28 & 0.10 & 9.98 & -9.88 & 14.9 & 40.9 & -26.0 & 2.55 & 1.75 & 0.80 & 20.5 & 15.0 & 5.49 & 30.8 & 41.1 & -10.3 \\
\hline 29 & 0.48 & 5.27 & -4.79 & 9.67 & 21.6 & -11.9 & 108 & 0.93 & 107.00 & 11.1 & 7.91 & 3.22 & 147 & 21.7 & 125 \\
\hline 30 & -0.11 & 4.05 & -4.16 & 6.46 & 16.6 & -10.1 & 1.55 & 0.71 & 0.84 & 8.58 & 6.07 & 2.51 & 36.2 & 16.7 & 19.5 \\
\hline 31 & -0.28 & 3.78 & -4.06 & 6.53 & 15.5 & -8.95 & 2.95 & 0.66 & 2.28 & 8.05 & 5.67 & 2.38 & 54.2 & 15.6 & 38.6 \\
\hline 32 & 0.41 & 17.2 & -16.8 & 18.9 & 70.5 & -51.6 & 7.10 & 3.02 & 4.08 & 47.1 & 25.8 & 21.3 & 66.1 & 70.9 & -4.75 \\
\hline 33 & 0.40 & 17.5 & -17.1 & 20.7 & 71.7 & -51.0 & 5.28 & 3.07 & 2.21 & 49.7 & 26.3 & 23.4 & 68.4 & 72.1 & -3.65 \\
\hline AVERAGE & 0.16 & 7.96 & -7.80 & 11.9 & 32.6 & -20.7 & 12.7 & 1.40 & 11.30 & 21.3 & 11.9 & 9.36 & 74.4 & 32.8 & 41.6 \\
\hline
\end{tabular}

\begin{tabular}{|c|c|c|c|c|c|c|c|c|c|c|c|c|c|c|c|}
\hline & Mo & Mo & Mo & $\mathbf{N i}$ & $\mathbf{N i}$ & $\mathbf{N i}$ & $\mathbf{P b}$ & $\mathbf{P b}$ & $\mathbf{P b}$ & $\mathbf{V}$ & $\mathbf{V}$ & V & $\mathbf{Z n}$ & Zn & Zn \\
\hline 34 & -1.67 & 2.46 & -4.13 & 20.9 & 10.1 & 10.8 & 1.07 & 0.432 & 0.554 & 16.8 & 3.69 & 13.1 & 60.4 & 10.1 & 50.3 \\
\hline 35 & -1.78 & 5.33 & -7.11 & 16.9 & 21.8 & -4.87 & -0.72 & 0.94 & -1.82 & 49.9 & 7.99 & 41.9 & 35.0 & 21.9 & 13.0 \\
\hline 36 & -1.33 & 5.02 & -6.35 & 14.1 & 20.6 & -6.42 & 26.7 & 0.88 & 25.7 & 14.9 & 7.53 & 7.40 & 140 & 20.7 & 119 \\
\hline 37 & -0.11 & 9.15 & -9.26 & 24.3 & 37.5 & -13.2 & 129 & 1.61 & 127 & 32.9 & 13.7 & 19.1 & 134 & 37.7 & 96.7 \\
\hline 38 & -0.795 & 4.47 & -5.26 & 17.2 & 18.3 & -1.11 & 1,770 & 0.78 & 1,770 & 13.5 & 6.70 & 6.80 & 472 & 18.4 & 454 \\
\hline 39 & 0.694 & 6.89 & -6.20 & 15.5 & 28.2 & -12.7 & 295 & 1.21 & 293 & 26.6 & 10.3 & 16.2 & 365 & 28.4 & 337 \\
\hline 40 & 0.453 & 6.31 & -5.85 & 14.2 & 25.8 & -11.6 & 11.0 & 1.11 & 9.69 & 25.5 & 9.45 & 16.0 & 394 & 26.0 & 368 \\
\hline AVERAGE & -0.649 & 5.66 & -6.31 & 17.6 & 23.2 & -5.59 & 318 & 0.99 & 317 & 25.7 & 8.49 & 17.2 & 229 & 23.3 & 205 \\
\hline
\end{tabular}

\begin{tabular}{|c|c|c|c|c|c|c|c|c|c|c|c|c|c|c|}
\hline CUMULATIVE & -0.153 & 7.07 & -7.22 & 14.1 & 28.9 & -14.8 & 132 & 1.24 & 130 & 23.0 & 10.6 & 12 & & 105 \\
\hline
\end{tabular}

